\title{
Analysis of switched-rigid floating oscillator
}

\author{
Prabhakar R. Marur \\ India Science Lab, General Motors R\&D, Creator Building, ITP, Bangalore - 560 066, India
}

Received 29 October 2007

Revised 2008

\begin{abstract}
In explicit finite element simulations, a technique called deformable-to-rigid (D2R) switching is used routinely to reduce the computation time. Using the $\mathrm{D} 2 \mathrm{R}$ option, the deformable parts in the model can be switched to rigid and reverted back to deformable when needed during the analysis. The time of activation of D2R however influences the overall dynamics of the system being analyzed. In this paper, a theoretical basis for the selection of time of rigid switching based on system energy is established. A floating oscillator problem is investigated for this purpose and closed-form analytical expressions are derived for different phases in rigid switching. The analytical expressions are validated by comparing the theoretical results with numerical computations.
\end{abstract}

Keywords: Deformable-rigid switching, rigid-body modes, impulse response, mechanical vibration

\section{Introduction}

In explicit finite element analysis, switching of parts that are executing largely rigid body motion to a rigid part during the analysis reduces the overall analysis time significantly. The D2R feature (e.g., *DEFORMABLE-TORIGID keyword in LS-Dyna [1]) can be used to advantage in automotive crash simulations. For example, in a rollover event, the vehicle is acted upon only by gravity once it looses contact with the tripping mechanism and the road surface [2]. Hence the vehicle can be treated as a collection of rigid bodies until the vehicle is about to make ground contact. At which time the vehicle could be switched back to deformable to compute the structural deformation due to ground impact.

As rigid body analysis requires computation of only the generalized displacements of the part center of gravity (CG), it eliminates the need for the computation of element-centered variables. This results in significant reduction in the computational effort required. The use of D2R switching option however requires a special attention to the time of exercising the D2R switch, so that the overall dynamics of the vehicle is not influenced by the switching.

To understand the mechanics of D2R switching, and get an insight into the physics of the problem, an instrument casing with a suspended mass is studied. The instrument package is modeled as a floating oscillator which is essentially a system of two unconstrained masses connected by a spring. The floating oscillator system is used for benchmarking bang-bang control [3] analysis, for simplifying vehicle crash analysis [4], for validating biofidelity testing [5], and for analyzing forced vibration of unconstrained systems [6,7].

The objective of this paper is to establish a theoretical basis for the selection of time of rigid switching based on the system energy. The time of switching to rigid and back should be such that the dynamic behavior of the system is not altered. A simple box model representing an automotive vehicle in rollover is modeled in LS-Dyna to illustrate the influence of rigid switching on the dynamic response of the system. To provide an insight into the physics of rigid switching, an analytical study of the floating oscillator is performed. Closed-form analytical expressions are derived for all the phases in rigid switching of the floating oscillator. An energy-based approach is proposed for the selection of the time of D2R switching, and the analytical results are validated using the finite element results obtained using the box model.

\footnotetext{
*Corresponding author. Tel.: +91 804118 4043; Fax: +1 509275 8715; E-mail: prabhakar.marur@gm.com.
} 


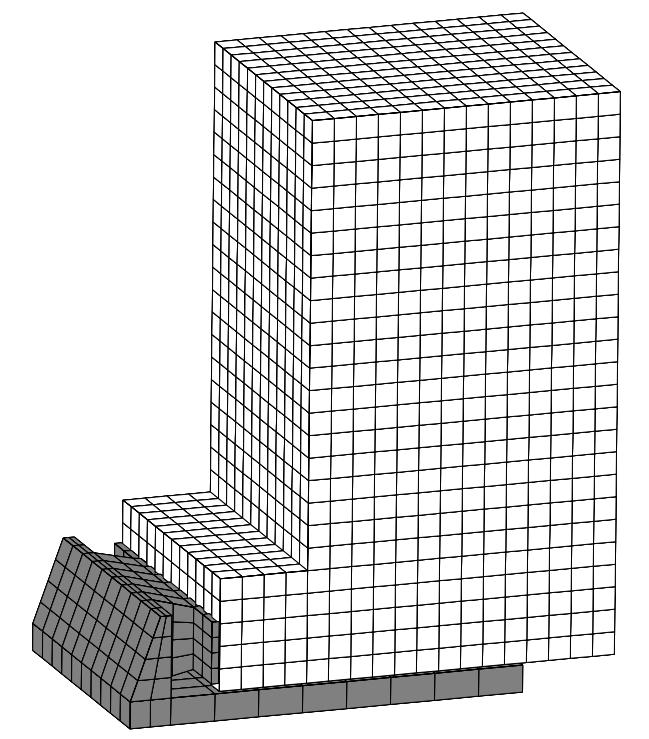

Fig. 1. Simple box model on a deceleration sled representing curb-tripped rollover testing.

\section{Influence of D2R Switching}

The influence of D2R switching on the overall dynamics of a system is demonstrated using a simple box model shown in Fig. 1. The model is an abstraction of the curb-tripped rollover of automotive vehicle. The rollover test is typically performed in the laboratory environment using a deceleration rollover sled [8]. The sled is essentially a moving platform that carries the vehicle and a set of brakes that are positioned along the track. The deceleration brakes are sequenced and tuned to apply desired deceleration pulse on the moving platform. Initially both the vehicle and the sled are moved together with the test velocity and subsequently the platform is decelerated. The vehicle, having only frictional contact with the platform, slides out and engages the side walls of the sled resulting in large lateral forces at the tire/wall interface, causing the vehicle to rollover.

The simplified model shown in Fig. 1 consists of a box structure with a step that comes into contact with the curb. The box model is supported on a sled with a honeycomb structure to cushion the impact of the box structure on the curb. Of the two parts that make up the box model, the stepped part remains deformable through the simulation, and the box is switched to rigid at time $t_{r}$. Using this simplified model, LS-Dyna simulations are performed applying same uniform velocity to all the nodes in the model and applying prescribed deceleration only to the sled, leading to rolling of the box. The numerical simulations are performed with and without D2R switching, and the computed CG position and roll angle time histories are compared in Fig. 2. It must be remarked that for the deformable body, the CG is taken to be the CG of the initial configuration. The simulation with D2R switching (referred to as D2R model in Fig. 2 and subsequent figures) predicts less roll compared to the reference model.

As the reference model and the D2R model differ only in one part being switched to rigid during simulation, it is plausible that the elastic energy in the system is responsible for the deviation in the computed results. The energy remains constant once parts are moved to the rigid, and the constant value depends on the time of D2R switching. If D2R switching is too soon then less energy is stored which leads to underestimation of roll angle, and if the switching results in higher energy levels than the reference model, then the roll angle is overestimated as shown in Fig. 2c.

To further illustrate the influence of the rigid switching on the overall dynamics and to enable analytical treatment of D2R switching, an instrument casing with a suspended mass is modeled in LS-Dyna as shown in Fig. 3. The spring is switched to rigid at $t_{r}$ and switched back to deformable state at time $t_{d}$. By choosing different $t_{r}$, the effect of switching on the vibratory motion of the suspended mass is demonstrated. The mass and the frame are modeled with solid elements in LS-Dyna, and these parts remain rigid through the analysis. The spring is modeled with brick elements as shown in Fig. 3, as discrete elements cannot be used in D2R switching. During the analysis only the part that represents the spring gets switched to rigid. Mass proportional damping is imposed in the model, and an 
a)

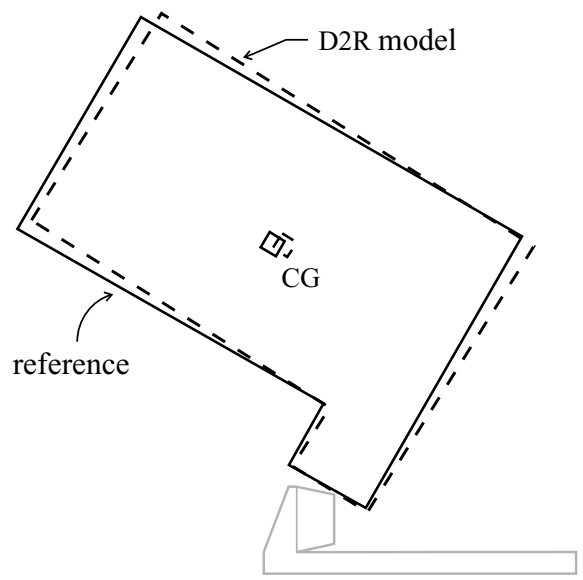

b)

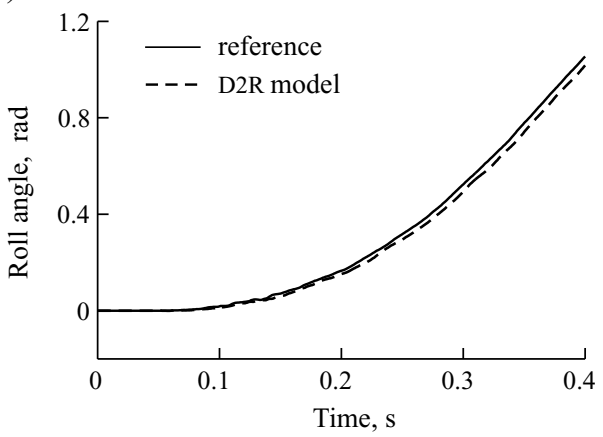

c)

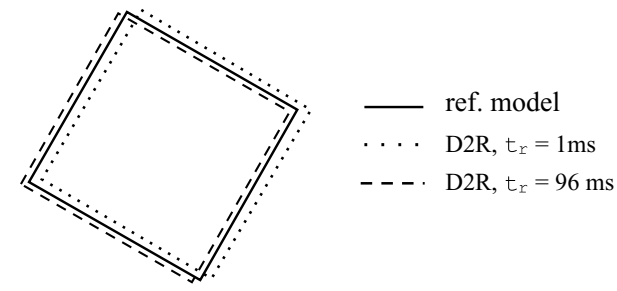

Fig. 2. a) Superposed edge plots of the box model with and without D2R switching. b) Comparison of roll angle of the box CG. The rigid switching is activated at $1 \mathrm{~ms}$. c) Overestimation and underestimation of roll angle illustrated by superposed edge plots of box CG.

initial of velocity of $6 \mathrm{~m} / \mathrm{s}$ in the vertical direction is applied to the frame. The vertical displacement history of the suspended mass computed using LS-Dyna is shown in Fig. 4 for different $t_{r}$ values. The variation in the vertical displacement after $t_{d}$ clearly demonstrates the effect of the choice of rigid switch timings.

\section{D2R Switching of Floating Oscillator}

The instrument model described in the previous section and shown in Fig. 3 is analyzed in this section to express the dependence of the system response on the time of switching analytically. Closed form analytical expressions are derived for the three different phases: (1) initial deformable state, (2) rigid phase and (3) 'spring back' stage. The equations of motion are different in different phases, and the end condition of one phase becomes the initial condition for the next. 


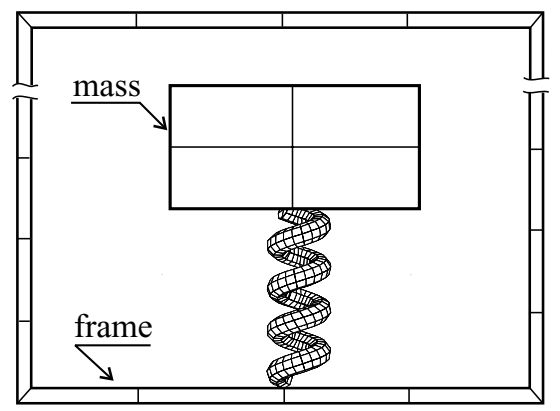

Fig. 3. FE mesh of the instrument casing with suspended mass.

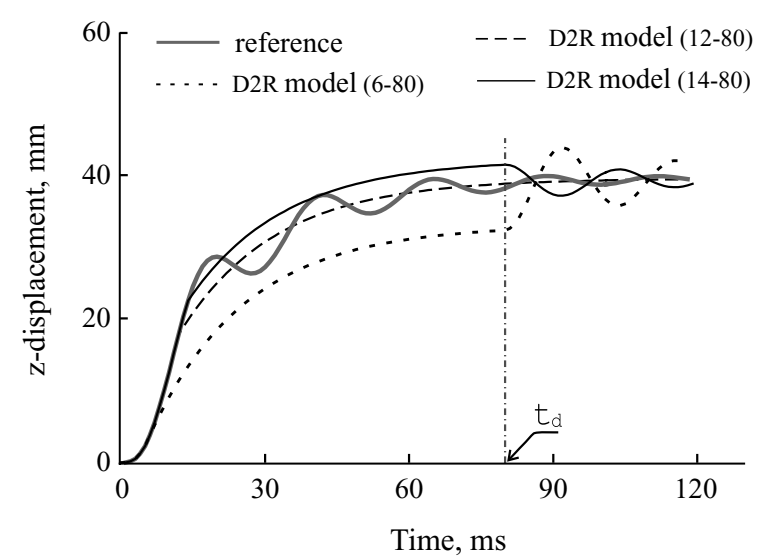

Fig. 4. Comparison of vertical displacement of the suspended mass for various D2R switch timings. The numbers in the parenthesis denote $t_{r}$ and $t_{d}$ respectively in this figure and subsequent figures.

a)

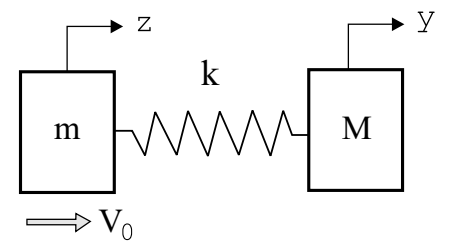

b)

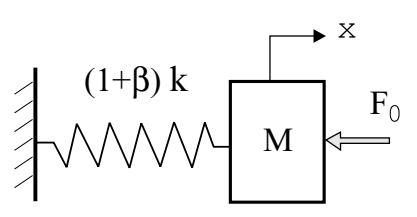

Fig. 5. a) 2-dof model of the instrument vibration. b) Equivalent single-dof system.

\subsection{Initial deformable state}

In the initial state, the instrument casing with the suspended mass is treated as a two degrees of freedom (dof) system as shown in Fig. 5a. The equations of motion for mass proportional damping can be given as

$$
\left[\begin{array}{lr}
m & 0 \\
0 & M
\end{array}\right]\left\{\begin{array}{l}
\ddot{z} \\
\ddot{y}
\end{array}\right\}+\alpha\left[\begin{array}{lr}
m & 0 \\
0 & M
\end{array}\right]\left\{\begin{array}{l}
\dot{z} \\
\dot{y}
\end{array}\right\}+k\left[\begin{array}{lr}
1 & -1 \\
-1 & 1
\end{array}\right]\left\{\begin{array}{l}
z \\
y
\end{array}\right\}=0,
$$

with initial conditions $z(0)=y(0)=\dot{y}(0)=0$, and $\dot{z}(0)=V_{0}$, where $V_{0}$ is the initial velocity imposed on the frame, $\alpha$ is mass proportional damping, $k$ is the spring stiffness, $M$ is the suspended mass and $m$ is the mass of the frame. The notation $(\dot{)}$ denotes differentiation of a variable with respect to time $t$.

Assuming harmonic motion of the form $z(t)=C_{1} e^{\lambda t}$ and $y(t)=C_{2} e^{\lambda t}$, the Eq. (1) can be cast as 


$$
\left[\begin{array}{lr}
m \lambda^{2}+\alpha m \lambda+k & -k \\
-k & M \lambda^{2}+\alpha M \lambda+k
\end{array}\right]\left\{\begin{array}{l}
C_{1} \\
C_{2}
\end{array}\right\}=0,
$$

where $C_{1}, C_{2}$ and $\lambda$ are undetermined constants.

By setting the determinant of the square matrix in the above equation to zero, the characteristic equation can be given as

$$
\lambda(\lambda+\alpha)\left[\lambda^{2}+\alpha \lambda+\omega_{n}^{2}(1+\beta)\right],=0
$$

where $\beta=M / m$ and $\omega_{n}=\sqrt{k / M}$.

By solving the characteristic polynomial, a rigid translation mode corresponding to $\lambda_{1}=0$ and $\lambda_{2}=-\alpha$, and an oscillatory mode corresponding to

$$
\lambda_{3,4}=-\frac{\alpha}{2} \pm i \sqrt{(1+\beta) \omega_{n}^{2}-\left(\frac{\alpha}{2}\right)^{2}}
$$

are obtained. The corresponding eigenvectors are given by $\left[\begin{array}{ll}1 & 1\end{array}\right]^{T}$ and $\left[\begin{array}{ll}-\beta & 1\end{array}\right]^{T}$. The mode shapes are obtained as

$$
\left(A_{1}+A_{2} e^{-\alpha t}\right) \text { and } e^{-\frac{\alpha t}{2}}\left(B_{1} \cos \omega_{2} t+B_{2} \sin \omega_{2} t\right)
$$

where $\omega_{2}=\sqrt{(1+\beta) \omega_{n}^{2}-\left(\frac{\alpha}{2}\right)^{2}}$, and $A$ 's, $B$ 's are undetermined coefficients.

The complete solution can be given as

$$
\left\{\begin{array}{l}
z(t) \\
y(t)
\end{array}\right\}=\left\{\begin{array}{l}
1 \\
1
\end{array}\right\}\left(A_{1}+A_{2} e^{-\alpha t}\right)-\left\{\begin{array}{c}
-\beta \\
1
\end{array}\right\} e^{-\alpha t / 2}\left(B_{1} \cos \omega_{2} t+B_{2} \sin \omega_{2} t\right)
$$

Imposing the initial conditions, the coefficients are evaluated as $A_{1}=-A_{2}, A_{2}=-V_{0} / \alpha(1+\beta), B_{1}=0$ and $B_{2}=-V_{0} / \omega_{2}(1+\beta)$. Substituting for these coefficients, the displacements of the masses in this phase can be obtained as

$$
\begin{aligned}
& z(t)=\frac{V_{0}}{1+\beta}\left[\frac{1}{\alpha}\left(1-e^{-\alpha t}\right)+\frac{\beta e^{-\alpha t / 2}}{\omega_{2}} \sin \omega_{2} t\right], \\
& y(t)=\frac{V_{0}}{1+\beta}\left[\frac{1}{\alpha}\left(1-e^{-\alpha t}\right)-\frac{e^{-\alpha t / 2}}{\omega_{2}} \sin \omega_{2} t\right] .
\end{aligned}
$$

\subsection{Rigid phase}

The rigid phase of the motion is reached when rigid switching is triggered at $t=t_{r}$. At this time the spring becomes rigid, hence both $m$ and $M$ must have a common velocity $V_{1}$. For conserving the total momentum before and after the D2R switching, $V_{1}$ must satisfy the following relation

$$
(m+M) V_{1}=m \dot{z}\left(t_{r}\right)+M \dot{y}\left(t_{r}\right),
$$

from which $V_{1}$ is obtained as $\left(\dot{z}_{t_{r}}+\beta \dot{y}_{t_{r}}\right) /(1+\beta)$.

Since mass proportional damping is active in the system, the combined rigid entity loses kinetic energy and decelerates. The differential equation to be solved in this phase is a first-order equation in $V(t)$ given by

$$
\frac{d V}{d t}+\alpha V=0
$$

with the initial condition given by $V\left(t_{r}\right)=V_{1}$. The solution of this ordinary differential equation is

$$
V(t)=A_{3} e^{-\alpha t},
$$

where $A_{3}$ is an undermined coefficient. The rigid body displacement $\delta(t)$ can be obtained as 
$\delta(t)=\left[1-e^{-\alpha t}\right] \frac{A_{3}}{\alpha}$.

Applying the initial condition at $t=t_{r}$, the displacements in the rigid phase are obtained as

$$
\begin{array}{ll}
z(t)=z\left(t_{r}\right)+\left[1-e^{-\alpha\left(t-t_{r}\right)}\right] \frac{V_{1}}{\alpha}, & t_{r} \leqslant t<t_{d}, \\
y(t)=y\left(t_{r}\right)+\left[1-e^{-\alpha\left(t-t_{r}\right)}\right] \frac{V_{1}}{\alpha}, & t_{r} \leqslant t<t_{d},
\end{array}
$$

where $t_{d}$ is the time at which deformable switching is enabled. In this phase, the velocities can be given as $\dot{z}=\dot{y}=V_{1} e^{-\alpha\left(t-t_{r}\right)}$.

\subsection{Spring-back phase}

The final phase is reached when the model is switched back to deformable at $t=t_{d}$. The spring that is 'frozen' at its displaced position at $t_{r}$ releases the internal energy. Although the total spring deformation denoted by $\Delta\left(=y\left(t_{r}\right)-z\left(t_{r}\right)\right)$ is known, it is not possible to impose initial displacement conditions on the individual masses in this phase.

The reason for the inability to ascertain the initial conditions for the individual masses at $t_{d}$ is briefly described here. From Eq. (12), one could obtain $z\left(t_{d}\right)$ and $y\left(t_{d}\right)$. However, these values are simply the location references for the masses, whereas the initial conditions for the masses are dependent on the deformation $\Delta$ that gets released at the time of spring-back. Although the net compression $\Delta$ released by the locked-in spring is known, apportioning the same into $\delta_{1}$ and $\delta_{2}$ as initial displacements for the two masses is not possible.

A simple solution to the problem of apportioning the total spring-back is sought by using the impulse force that gets released at the time of switching. Since the force has to be the same on both the masses, while the displacements need not, this approach is followed in this work. The solution process is simplified further, as the nature of the motion of the system is known from the solution derived in the initial deformable phase. As the masses move as a rigid system with the same velocity, only the oscillatory mode needs to be computed. From the equations derived in the initial deformable phase, the rigid body motion is known and the same is given by

$$
\frac{V_{2}}{\alpha}\left[1-e^{-\alpha\left(t-t_{d}\right)}\right],
$$

where $V_{2}$ is the velocity of the combined masses at $t_{d}$. The oscillatory motion is obtained by solving an equivalent single-dof system. Superposition of these two solutions gives the complete solution.

The oscillatory motion is described using an equivalent single-dof system with a spring stiffness $(1+\beta) k$ and the mass $M$ as shown in Figure 5b. The equation of motion for the single-dof system under mass proportional damping can be given as

$$
\ddot{x}+\alpha \dot{x}+(1+\beta) \frac{k}{M}=\frac{F_{0}}{M},
$$

where $F_{0}$ is the imposed impulse force equaling $k \Delta$, and $x$ is the generalized coordinate of the equivalent single-dof system.

The complimentary function is obtained as

$$
x=e^{-\alpha t / 2}\left(A_{4} \cos \omega_{2} t+B_{4} \sin \omega_{2} t\right),
$$

where $A_{4}$ and $B_{4}$ are undetermined coefficients, and the particular integral can be given as $F_{0} /[(1+\beta) k]$.

The complete solution can be given as

$$
x=e^{-\alpha t / 2}\left(A_{4} \cos \omega_{2} t+B_{4} \sin \omega_{2} t\right)+\frac{\Delta}{1+\beta} .
$$

The initial conditions for the equivalent single-dof can be taken as $x(0)=\dot{x}(0)=0$ as the relative displacement and velocity of the masses are identically zero. Imposing the initial conditions, the expression for $x$ can be obtained as 


$$
x=\frac{\Delta}{1+\beta}\left[1-e^{-\alpha t / 2}\left(\cos \omega_{2} t+\frac{\alpha}{2 \omega_{2}} \sin \omega_{2} t\right)\right] .
$$

The individual modal values for the original system can be obtained from $x$ using the eigenvector as $\left[\begin{array}{ll}z & y\end{array}\right]^{T}=$ $x\left[\begin{array}{ll}-\beta & 1\end{array}\right]^{T}$. Finally, the displacements of the masses of the original system for $t>t_{d}$ can be given as

$$
\begin{aligned}
z(t)= & z\left(t_{d}\right)+\frac{V_{2}}{\alpha}\left[1-e^{-\alpha\left(t-t_{d}\right)}\right] \\
& +\frac{\beta \Delta}{1+\beta}\left[1-e^{-\alpha\left(t-t_{d}\right) / 2}\left(\cos \omega_{2}\left(t-t_{d}\right)+\frac{\alpha}{2 \omega_{2}} \sin \omega_{2}\left(t-t_{d}\right)\right)\right], \\
y(t)= & y\left(t_{d}\right)+\frac{V_{2}}{\alpha}\left[1-e^{-\alpha\left(t-t_{d}\right)}\right] \\
& -\frac{\Delta}{1+\beta}\left[1-e^{-\alpha\left(t-t_{d}\right) / 2}\left(\cos \omega_{2}\left(t-t_{d}\right)+\frac{\alpha}{2 \omega_{2}} \sin \omega_{2}\left(t-t_{d}\right)\right)\right] .
\end{aligned}
$$

\subsection{Comparison with numerical results}

The analytical expressions derived in the previous section are validated against numerical results obtained with LS-Dyna simulations. The displacement and velocity time histories of the frame and the suspended mass are computed using the model data of $k=660 \mathrm{kN} / \mathrm{m}, M=31.4 \mathrm{~kg}, \beta=2$ and $\alpha=50$. The initial velocity imposed on the frame is $6 \mathrm{~ms}^{-1}$. The D2R switching is triggered at $t=6 \mathrm{~ms}$ and deactivated at $t=80 \mathrm{~ms}$. The analytical results obtained for the specified input data are compared with those obtained with LS-Dyna in Fig. 6. The displacements of the masses computed by the analytical model are in close agreement with the numerical computation as can be noted in Figs 6a and 6b. In the case of velocities, analytical results closely match the LS-Dyna results barring the sharp transients at rigid switching times, which is an artifact of the explicit finite element solution scheme used.

\section{Energy-based approach to D2R switching}

From the analytical expressions derived in the previous section, it clear that the deformations are 'locked-in' at the time of switching to rigid, and an equivalent elastic energy gets released when the parts are set to deformable. Hence the ensuing motion of a part is dependent on the energy that gets released at the time of switch back. Hence the choice of $t_{r}$ and $t_{d}$ must be such that the energy that needs to be released at $t_{d}$ must be 'locked-in' at the time of rigid switching. As a result the analyst must first decide the time of switch back.

As an illustration of this approach, let $64 \mathrm{~ms}$ be the time of switching back to deformable. A horizontal line corresponding to the energy level at $64 \mathrm{~ms}$ can be drawn in the internal energy plot as shown in Fig. 7a. The intersection of this line with the energy curve gives the choice of D2R timings. In this example, time of D2R switching is chosen as $13 \mathrm{~ms}$. Using these timings, analytical results computed using the expressions given in Section 3. The vertical displacement of the suspended mass obtained using the analytical model is compared with the reference results in Fig. 7b. The figure shows that the analytical computations agree well with the reference model in the time durations $0 \leqslant t \leqslant t_{r}$ and $t>t_{d}$ as desired.

Although this approach requires the knowledge from a previous run to determine $t_{r}$, it is not a limitation in the application of this technique. In rollover simulation, the typical parametric study involves changing parameters such as headroom in the vehicle, seat belt tension, roof strength, etc. The parametric variation amounts to several dozens of simulation runs, however, only one reference simulation is required for prescribing $t_{r}$, as these parameters do not influence the choice of $t_{r}$. 
a) Displacement of mass

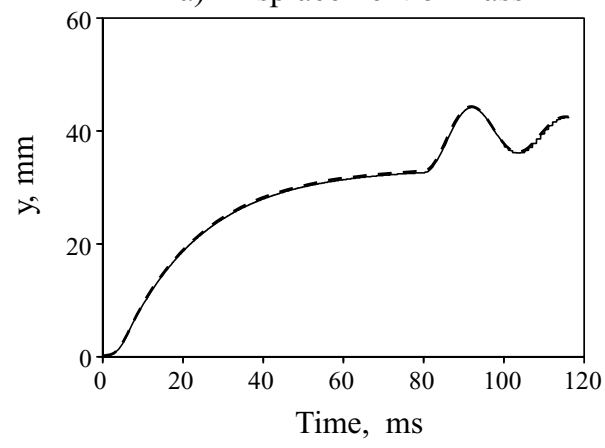

c) Velocity of mass

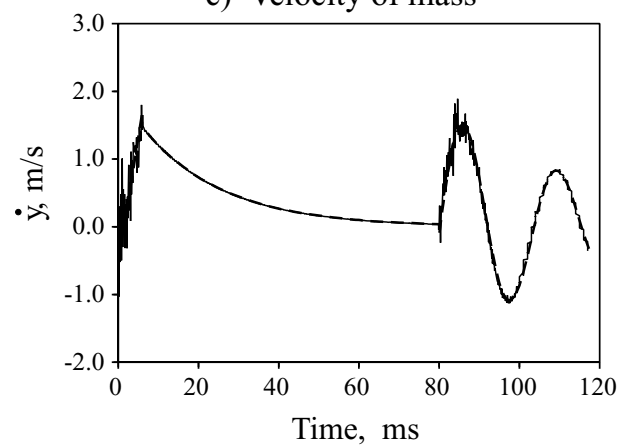

b) Displacement of frame

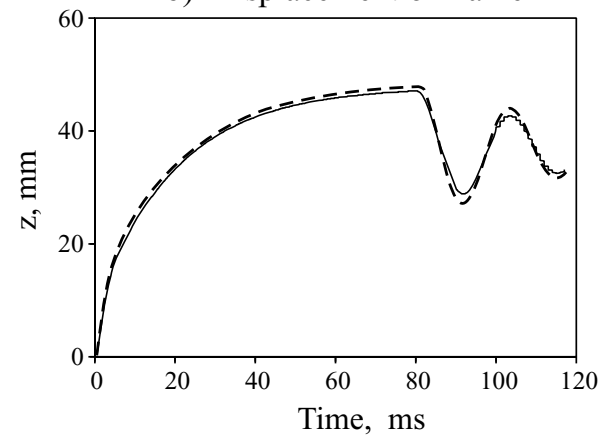

d) Velocity of frame

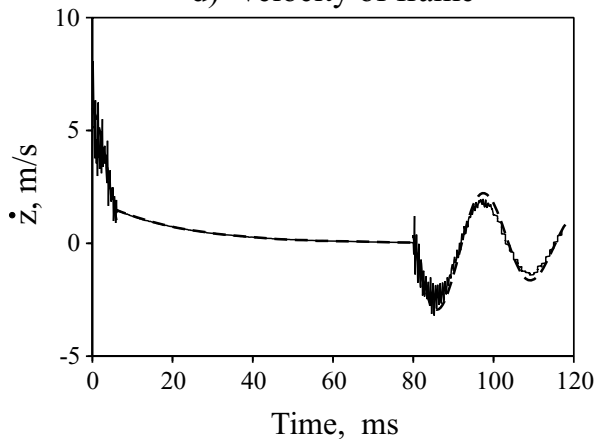

Fig. 6. Comparison of analytical computation of displacements and velocities with numerical results. —— LS-Dyna, --- analytical.

a)

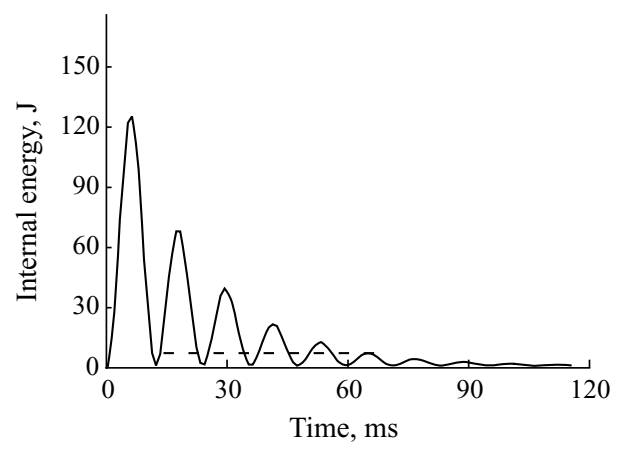

b)

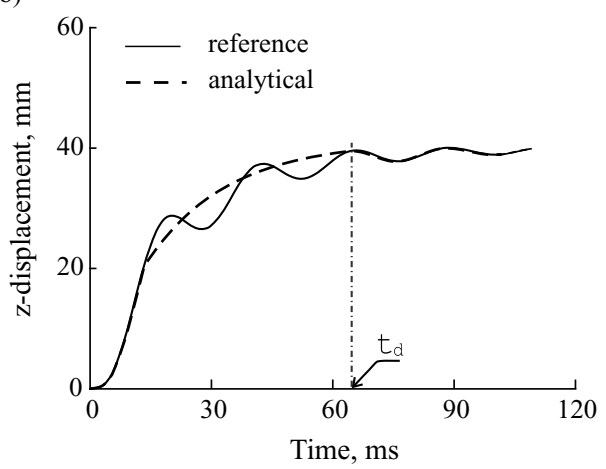

Fig. 7. a) Internal energy time history of the spring. b) Vertical displacement of the suspended mass.

\section{FE simulation with revised $D 2 R$ switching}

The finite element analysis of the box rollover model shown in Figure 1 is simulated again with the revised switch timings obtained using the energy criterion. The box model rolls and impacts the ground about $380 \mathrm{~ms}$, hence $t_{d}$ is taken as $370 \mathrm{~ms}$. In the internal energy plot of the box from the reference run, $t_{r}=70 \mathrm{~ms}$ has same internal energy level as at $t_{d}$. Using these revised switch timings, the LS-Dyna simulation is performed. The vertical displacement and roll angle history of the CG of the box are compared in Fig. 8. As can be noted in the figure the results obtained with D2R switching agree closely with the reference model.

This D2R switching technique is applied to curb trip rollover analysis of a FE model of a pick-up truck. The curb trip rollover is simulated using an open source FE model of Chevrolet C2500 pickup truck that can be obtained 
a)

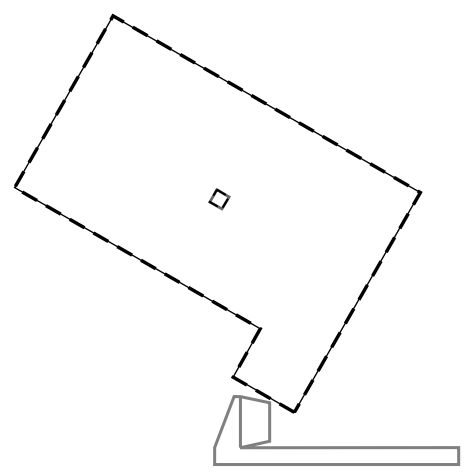

b)

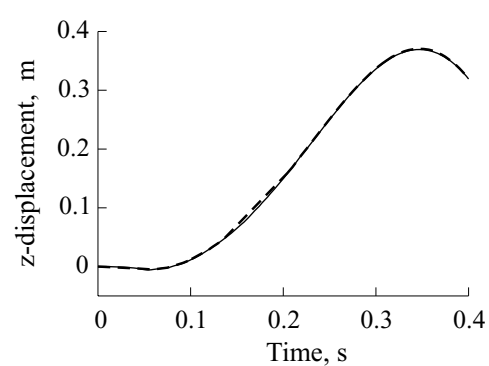

c)

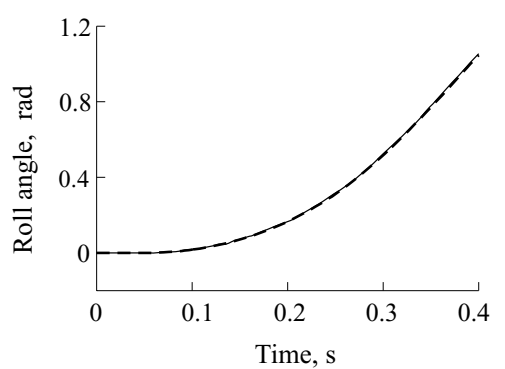

Fig. 8. a) Superposed edge plots of box CG with and without D2R switching. b) Comparison of vertical displacement of the CG. c) Comparison of roll angles. — reference, $---\mathrm{D} 2 \mathrm{R}$ model (70-370).

from National Crash Analysis Center [9]. The details of setting up curb trip rollover simulation are discussed in Ref. [2]. For the baseline simulation, the total CPU time required for the execution is 25.5 hours on a 8-CPU high performance computer. Using the $\mathrm{D} 2 \mathrm{R}$ approach, the simulation required only 6.5 hours on the same computer which is 75 percent less than the time required for executing the reference simulation.

\section{Conclusions}

The influence of the time of D2R switching on the overall dynamics of a system is studied using a floating oscillator. With a mass damped 2-dof model of the floating oscillator, closed form analytical expressions are derived for D2R switching and the physics behind the energy based rigid switching is investigated. It is concluded that the choice of timing of D2R switching should ensure that the energy stored in the system is same as that in the reference simulation at the time of switching back to deformable. A simplified box model representing automotive vehicle rollover is simulated using an explicit finite element code, and demonstrated that with switch timings chosen based on the energy criterion, the simulation results obtained with D2R switching agree well with the reference data. 


\section{References}

[1] LS-DYNA Keyword Users Manual Version 970, Livermore Software Technology Corporation, 2003.

[2] P.R. Marur and S. Namdeo, Techniques for reducing computational time in vehicle rollover simulations, International Journal of Vehicle Safety 2 (2008), 441-456.

[3] K. Fuwa, T. Narikiyo and Y. Funahashi, Construction of a Control System for Disturbance Rejection Using a Dual Observer, Electrical Engineering in Japan 138 (2002), 50-60.

[4] Y. Motozawa, US Patent No. 6578894.

[5] A.K. Roberts, R.W. Lowne, M. Beusenberg and D Cesari, Test procedure for defining biofidelity targets for lateral impact of test dummies, 13th ESV Conference, Paris, France, Paper 91-S8-W-18, 1991.

[6] L. Cveticanin, The Motion of a Two-Mass System With Non-Linear Connection, Journal of Sound and Vibration 252 (2002), $361-369$.

[7] L. Cveticanin, Vibrations of a free two-mass system with quadratic non-linearity and a constant excitation force, Journal of Sound and Vibration 270 (2004), 441-449.

[8] M. Rossey, Test Method for Simulating Vehicle Rollover, SAE Paper 2001-01-0475, 2001.

[9] NCAC (n.d.) FHWA/NHTSA National Crash Analysis Center, Model Retrieved on March 2006, available at: www.ncac.gwu.edu. 

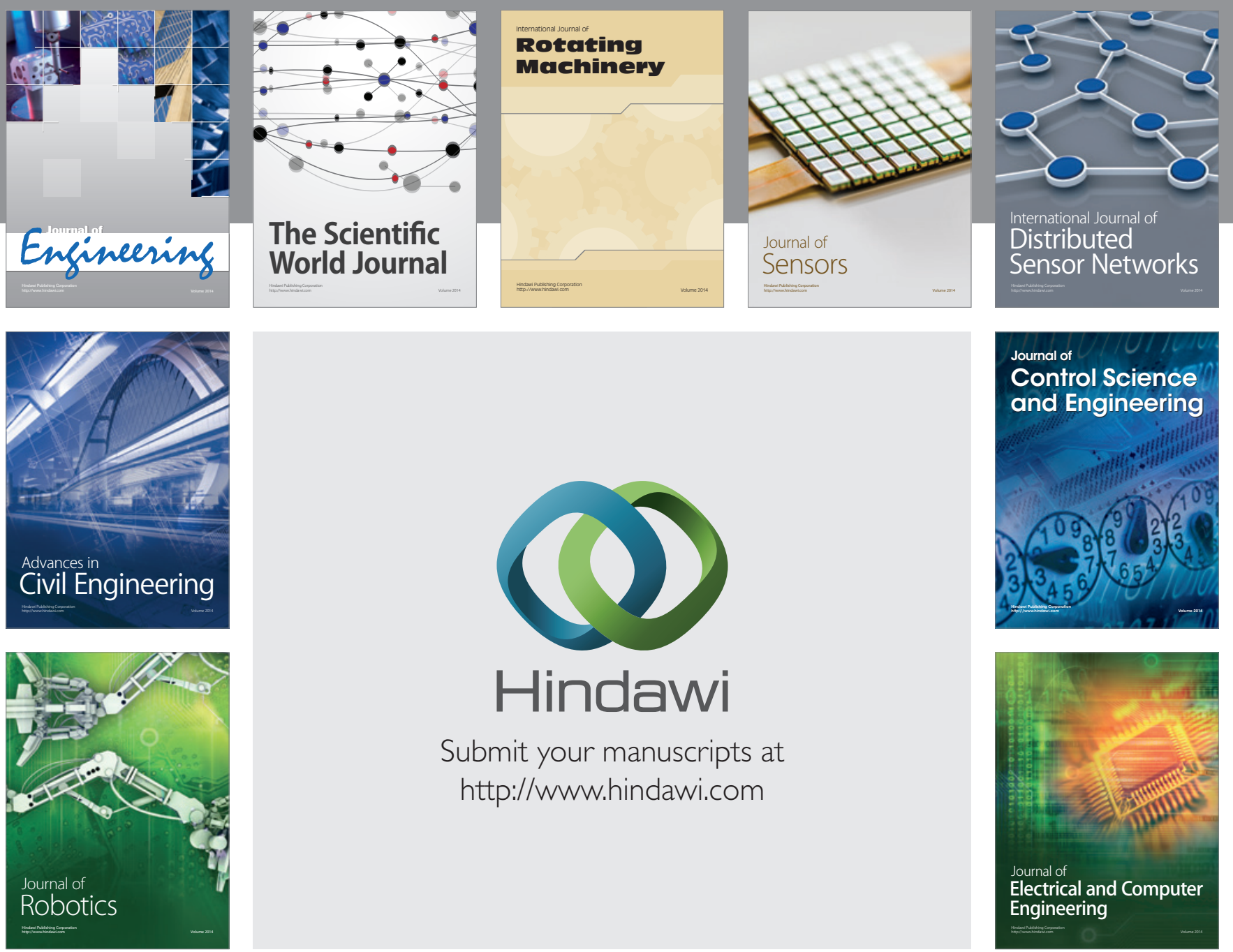

Submit your manuscripts at

http://www.hindawi.com
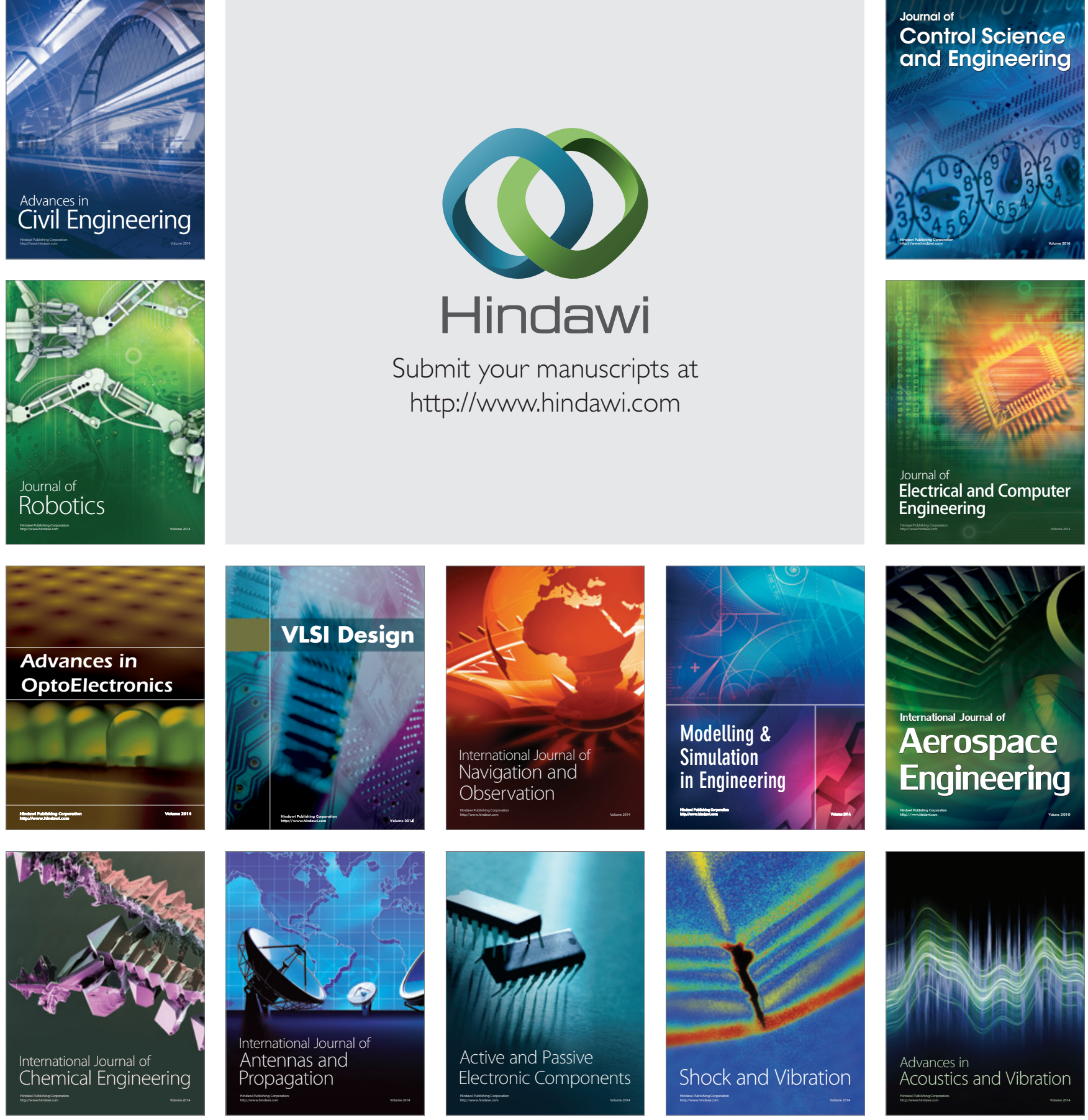